\title{
Neural Basis of Language: An Overview of An Evolving Model
}

\author{
Masazumi FuJII, ${ }^{1}$ Satoshi MAESAWA, ${ }^{2,3}$ Sumio ISHIAI, ${ }^{4}$ Kenichiro IWAMI, ${ }^{1}$ \\ Miyako FUTAMURA, ${ }^{5}$ and Kiyoshi SAITO ${ }^{1}$
}

\author{
${ }^{1}$ Department of Neurosurgery, Fukushima Medical University, Fukushima, Fukushima; \\ ${ }^{2}$ Brain and Mind Research Center, Nagoya University, Nagoya, Aichi; \\ ${ }^{3}$ Department of Neurosurgery, Nagoya University Graduate School of Medicine, Nagoya, Aichi; \\ ${ }^{4}$ Department of Rehabilitation Medicine, Sapporo Medical University School \\ of Medicine, Sapporo, Hokkaido; \\ ${ }^{5}$ Rehabilitation Center, Fukushima Medical University, Fukushima, Fukushima
}

\begin{abstract}
The neural basis of language had been considered as a simple model consisting of the Broca's area, the Wernicke's area, and the arcuate fasciculus (AF) connecting the above two cortical areas. However, it has grown to a larger and more complex model based upon recent advancements in neuroscience such as precise imaging studies of aphasic patients, diffusion tensor imaging studies, functional magnetic resonance imaging studies, and electrophysiological studies with cortical and subcortical stimulation during awake surgery. In the present model, language is considered to be processed through two distinct pathways, the dorsal stream and the ventral stream. The core of the dorsal stream is the superior longitudinal fasciculus/ $\mathrm{AF}$, which is mainly associated with phonological processing. On the other hand, semantic processing is done mainly with the ventral stream consisting of the inferior fronto-occipital fasciculus and the intratemporal networks. The frontal aslant tract has recently been named the deep frontal tract connecting the supplementary motor area and the Broca's area and it plays an important role in driving and initiating speech. It is necessary for every neurosurgeon to have basic knowledge of the neural basis of language. This knowledge is essential to plan safer surgery and preserve the above neural structures during surgery.
\end{abstract}

Key words: neural basis of language, awake surgery, hodotopy

\section{Introduction}

Since Brodmann ${ }^{1)}$ and Penfield et al. ${ }^{2)}$ published their studies, the localizationistic view of the brain has been overwhelming. It was hitherto thought that each brain function is rigidly localized in a certain cortical area of the brain, and an injury to a certain area of the cortex would lead to a loss of corresponding brain function. In this view, cortices or cortical areas where neurons are located are more highlighted when considering the functional aspects of the brain, rather than white matter. However, recent advancements in neuroscience have suggested that the brain is organized in a complex network of multiple distinct neural circuits, ${ }^{3)}$ and works more like a dynamic network with plasticity. "Hodotopy" or the "hodotopic frame work" is a new concept proposed by Catani et al., ${ }^{4)}$ which includes both topological (cortical functional

Received: January 14, 2016; Accepted: March 16, 2016 epicenters) and hodological (connectivity between areas) views for understanding a particular brain function and its neural basis. In this framework, the brain, inside which the gray and white matters are part of the same interactive process, is organized as complex, multimodal, and integrated parallel distributed networks, with a cognitive process consisting of a continuous and often redundant stream of information dynamically modulated by experience and external environment. ${ }^{3)}$ When considering the neural basis of a function or dysfunction, especially cognitive functions such as language, it is important to focus on not only cortices but also on both cortices and white matter as a network.

Attitudes toward interpretation of the neural basis are different between the conventional topological approach and hodological/hodotopic approach, especially in the study of patients with a certain cognitive symptom. Each patient with the same dysfunction does not necessarily have an identical lesion, 
but demonstrates variation in the involved area. In the topological approach, the commonly injured cortical area among the patients with certain cognitive dysfunction is considered as the center of the function. ${ }^{5}$ In the hodological approach, on the other hand, because the function is considered as a representation of a network, each patient has a certain injury in a part of network of the function. ${ }^{6)}$ There is a good example demonstrating modern interpretation of a part of neural basis of language. Dronkers et al. reported a re-study of Paul Broca's historical cases with magnetic resonance imagings (MRIs) of the original patients' brains. They found that the lesions extended far deeper than the cortical areas that Broca was able to report and that the superior longitudinal fasciculus (SLF), which is a major association fiber tract connecting the frontal lobe and parietal lobe, was involved. ${ }^{7)}$ In fact, it is now known that injury of merely the cortical area of Broca's speech center does not cause "Broca's aphasia," but causes only transient speech disturbance or a more moderate form of aphasia. ${ }^{89}$ Broca's aphasia occurs when injury involves wider cortical areas than the Broca area, including the middle-inferior part of the precentral gyrus (PreCG) and the white matter underneath those areas. ${ }^{9,10)}$

This article attempts to describe the neural basis of language as a simple model based on recent advancements in neuroscience such as precise imaging studies of aphasic patients, diffusion tensor imaging studies, functional MRI studies, fiber dissection studies, and electrophysiological studies with cortical and subcortical stimulation during awake surgery, especially focusing on the white matter pathways. Even though the neural basis of language is not yet fully understood and is apparently not as simple as detailed in this article, basic knowledge of the same is still vital for neurosurgeons, since it could help to preserve patients' language function and enable further contributions to neuroscience.

\section{The Neural Basis of Language}

The classical model of neural basis of language consists of the Broca's area (the motor speech center), the Wernicke's area (the sensory speech center), and the arcuate fasciculus (AF) connecting the above two cortical areas. However, the model has grown to a larger and more complex one based upon recent advancements in neuroscience, comprising the frontal, temporal, and parietal language areas. The terms "Broca's area" and "Wernicke's area" are still used worldwide in many literatures, although it is known today that they are not the sole and definite language centers of the frontal or temporal lobe. Therefore, we have used the same terms to designate certain cortical areas as defined in this article. Broca's area is the pars triangularis and opercularis of the inferior frontal gyrus (IFG), corresponding to the Brodmann area 45 and 44, respectively. Wernicke's area is defined as the cortical area of the posterior STG and a part of the SMG, corresponding to the Brodmann area 22. The present model consists of two major pathways, the dorsal stream and the ventral stream ${ }^{11)}$ (Fig. 1). The dorsal stream is supported mainly by the SLF/AF and associated with phonological processing. ${ }^{12)}$ On the other hand, semantic processing occurs through the ventral stream supported mainly by the inferior fronto-occipital fasciculus (IFOF). ${ }^{12)}$ Briefly, one of the possible and reliable models based on the present neuroscience is described below in accordance with the

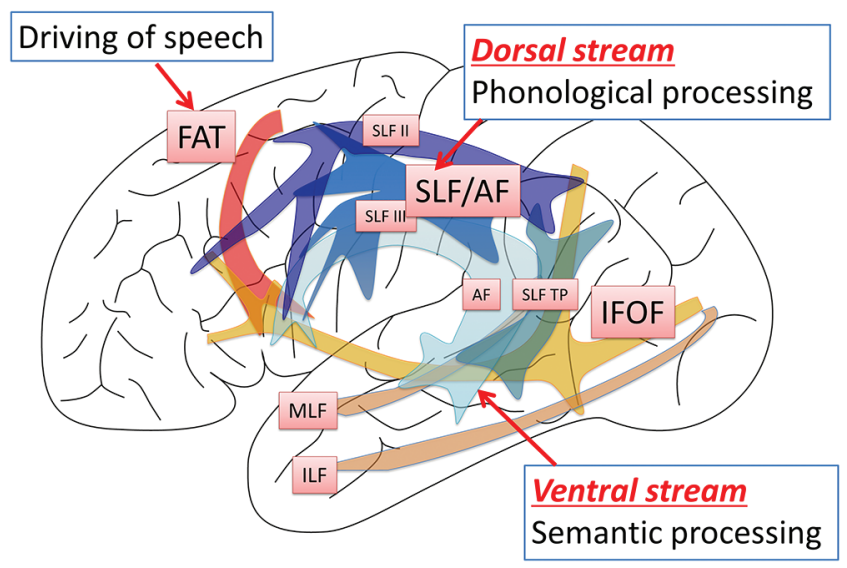

Fig. 1 Dual stream model of neural basis of language. The neural basis of language has been evolving to a more complex model with involvement of many cortical areas and white matter tracts than the classical model simply consisting of the Broca and Wernicke centers and the arcuate fasciculus (AF) connecting the above two. In the dual stream model proposed by Hickok et al., ${ }^{11)}$ the major network of language processing consists of two streams, the dorsal and ventral streams in the dominant hemisphere. The dorsal stream is associated with phonological processing via the superior longitudinal fasciculus (SLF) as a major trunk of the network. The SLF/AF consists of several subcomponents in two layers, the SLF II, the SLF III, and the SLF temporo-parietal (SLF TP) in the superficial layer and the classical AF in the deep layer. ${ }^{6,39,40)}$ The ventral stream is associated with semantic processing. It is assumed that the network consists of intra-temporal network, such as the middle longitudinal fasciculus (MLF) ${ }^{27,28)}$ and the inferior longitudinal fasciculus (ILF), ${ }^{29)}$ and the inferior fronto-occipital fasciculus (IFOF) ${ }^{33)}$ as an inter-lobe network. Adding to the dural stream model, there is another system inside the frontal lobe for "driving of speech." Recently named the frontal aslant tract (FAT) ${ }^{46,47)}$ is probably associated with initiation and spontaneity of speech. 
steps of functional aspects of language from perception to expression. Indeed, an electrophysiological study of the Broca's area demonstrated a series of language processing, lexical, grammatical, and phonological information that occurred in a sequential manner within a time window of $450 \mathrm{~ms} .{ }^{13)}$ However, please note and understand that the stepwise processes described expediently is only a one-sided view of language and may occur not only sequentially, but also simultaneously, with dynamic interactions in many cortical areas, even bilateral hemispheres, in reality. ${ }^{14-16)}$ In this article, only spoken language will be focused on without mentioning written language function, i.e., reading and writing.

\section{From sound to phoneme}

The first step of the speech comprehension is the conversion of the sound of language into phonemes, followed by storing them over a short period of time. This step is processed in the subnetwork including the superior temporal gyrus (STG), which is located adjacent to the Heschl's gyrus, namely the primary auditory area ${ }^{17,18)}$ (Fig. 2A). This sub-network might be supported by rostral and caudal streams originating from the primary auditory cortex, i.e., the Heschl's gyrus, in the superior temporal gyrus. ${ }^{19)}$ This area is also associated with "auditory short term memory" that performs as one of the subsystems of the working memory ${ }^{20)}$ The phonological process also occurs in association with the PreCG via the AF/SLF ${ }^{21)}$ and the Broca's area. ${ }^{22)}$ Injury of this subsystem gives rise to pure word deafness (word sound deafness), which is a specific disturbance of discrimination of the word sounds. ${ }^{23)}$ Patients with pure word deafness, however, are able to communicate through reading and writing. Recent studies with functional MRI suggest that voice perception and processing of phonemes occurs in the bilateral hemisphere. ${ }^{15,24)}$

2. Access to lexicon and semantic process of words The series of phonemes perceived in the above system are transferred to the middle temporal gyrus, accessing the word/lexicon, and meaning of the word $^{18,25)}$ (Fig. 2B). The posterior part of the middle temporal gyrus (MTG) is involved in accessing the lexicon, associating with not only auditory comprehension but also with expression of word by selecting word and phonemic processing of voice encoding via the $\mathrm{AF}^{2}{ }^{26)}$ The anterior part of the MTG is more involved in the semantic processing of words and selecting of specific words (lexical retrieval). The middle longitudinal fasciculus (MLF), ${ }^{27,28)}$ the inferior longitudinal fasciculus (ILF), ${ }^{29)}$ and the IFOF, ${ }^{12)}$ as well as the $U$ fibers and other short association fibers inside the temporal lobe are the components of network infrastructure. Intra-temporal network is associated with both phonological and semantic process of words. The lexical process, however, is not completed only in the temporal lobe. Even word-level processing is also integrated into the network described below.

3. Complex semantic process of sentence and context

More complex semantic process above the word level, such as sentences and context, takes place in many cortical areas, temporal lobe except for the STG, the inferior parietal lobule (IPL), and the IFG. The complex process includes process of syntax, semantic process of context, and selection of words in association with semantic memory. Such a large network among multiple cortical areas is probably supported by the IFOF, ${ }^{18,30-32)}$ which is one of the longest tracts in the human brain, connecting the above cortical areas (Fig. 2B). The IFOF is known to have two layers. ${ }^{33)}$ The superficial layer is considered to connect the superior parietal lobule, the occipital extrastriate cortex, the Wernicke's area, and the fusiform area at the occipito-temporal junction to the IFG (the pars orbitaris and triangularis), all of which are likely associated with the process of language ${ }^{33)}$ (Fig. 1). The deep layer originates in areas similar to those described above and terminates in wider areas of the frontal lobe, namely the orbitofrontal cortices, the middle frontal gyrus, and the dorsolateral prefrontal cortex, and is probably associated with language and other cognitive functions. ${ }^{33)}$ The IFG, as well as the MFG (lateral prefrontal cortex; LPFC), are involved especially in the process of syntax and enhancement of efficiency of semantic process of sentences. ${ }^{13,14,30,34)}$ Kinno et al. ${ }^{14)}$ identified three syntax-related networks and reorganization of them in glioma patients with agrammatic comprehension, suggesting that there is, in fact, redundancy or plasticity with multiple subnetworks in the syntax processing. Interestingly, the IFG or MFG (LPFC) was associated with all three networks and one of them involves participation of the contralateral hemisphere. As for the IPL, the angular gyrus is probably associated with semantic process ${ }^{35)}$ whereas the supra marginal gyrus (SMG) is more involved in phonological process. ${ }^{36,37)}$

\section{Phonological processing}

Phonological process is mainly done with the dorsal stream in which the frontal, parietal, and temporal lobe are all connected by the SLF/AF ${ }^{11,12,38)}$ (Fig. 2C). Conversion from the sound of voice into phoneme, lexical retrieval, repetition, and articulation are associated with this stream. The SLF/AF consists of several subcomponents in two layers, the 

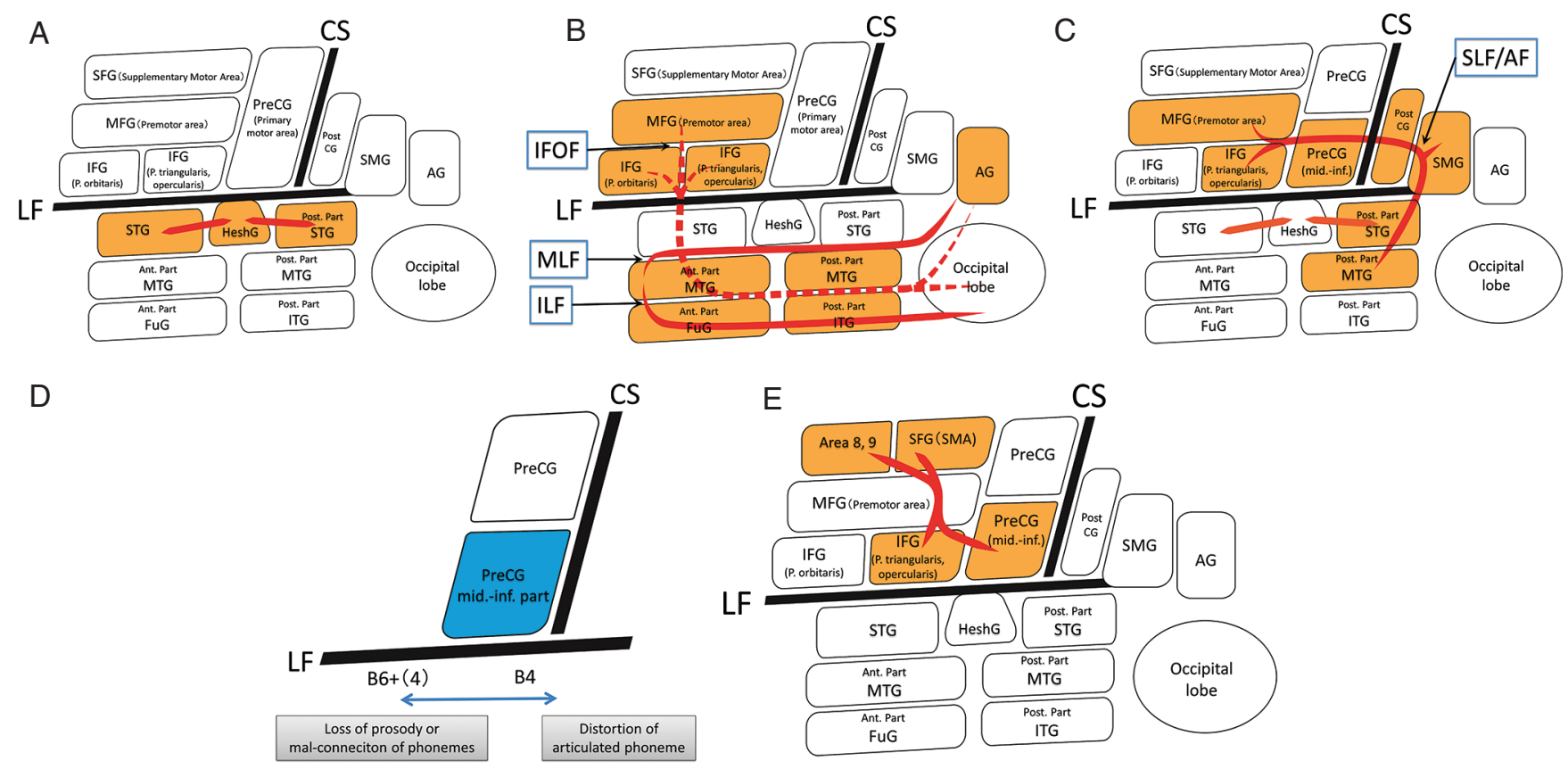

Fig. 2 A-E: Illustrations of neural basis of language networks, left hemisphere, and lateral view. A: From sound to phoneme. The first step of auditory comprehension of speech, conversion of the sound of language into phonemes, is located in the STG adjacent to the Heschl's G, namely the primary auditory area..$^{17,18)}$ This area is also associated with "auditory short-term memory" that played as one of the subsystems of the working memory. ${ }^{20)}$ This network is mainly supported by the U fibers among the area. B: Access to lexicon and semantic processing. The series of phonemes perceived in the above system are transferred to the MTG, accessing the word/lexicon and meaning of the word. The middle longitudinal fasciculus (MLF), ${ }^{27,28)}$ the inferior longitudinal fasciculus (ILF), ${ }^{29)}$ and the inferior fronto-occipital fasciculus (IFOF), ${ }^{33)}$ as well as the $\mathrm{U}$ fibers and other short association fibers inside the temporal lobe are the candidates of network infrastructure. More complex semantic process above word level, such as sentences and context takes place in many cortical areas, temporal lobe except for the STG, the inferior parietal lobule (IPL), and the inferior frontal gyrus (IFG). Such a large network among multiple cortical areas is probably supported by the IFOF, which is one of the longest tracts in human brain and connects the above cortical areas. ${ }^{18,30-32)} \mathrm{C}$ : Phonological processing. Phonological processing is mainly done with the dorsal stream in which the frontal, parietal, and temporal lobe are all connected by the SLF/AF. ${ }^{11,12,38)}$ Conversion from the sound of voice into phoneme, lexical retrieval, repetition, and articulation are associated with this stream. The SMG, PostCG, and the posterior part of STG connected with SLF/AF located under these cortices are profoundly associated phonological processes. ${ }^{20,36)}$ D: Articulation. The middle-inferior part of the dominant PreCG and its subcortical area is associated with articulation and its damage result in speech apraxia or, in other words, anarthria, in which the patients have symptoms such as loss of prosody, i.e., mal-connection of phonemes and distortion of phoneme. Damage in the anterior part of PreCG (Broadman area 6 and 4) tends to enhance loss of prosody or mal-connection of phonemes more, whereas damage in the posterior part of the PreCG (Broadman area 4) tends to cause distortion of phonemes. ${ }^{43)}$ E: Driving of speech. There is a pathway for initiation/driving of speech. The supplementary motor area and the medial aspect of the frontal lobe have been known to be associated with spontaneity and initiation of speech. ${ }^{44)}$ An intra-frontal network connecting between the medial aspect of the frontal lobe and the IFG and the middle-inferior part of PreCG, recently named as the frontal aslant tract (FAT) is suggested to have strong association with speech function. ${ }^{46-48)}$. AF: arcuate fasciculus, AG: angular gyrus, Ant.: anterior, FAT: frontal aslant tract, FuG: fusiform gyrus, Heschl G: Heschl gyrus, IFG: inferior fronta gyrus, IFOF: inferior fronto-occipital fasciculus, ITG: inferior temporal gyrus, MFG: middle frontal gyrus, mid.-inf.: PreCG middle and inferior part of precentral gyrus, MTG: middle temporal gyrus, PreCG: precentral gyrus, PostCG: postcentral gyrus, SFG: superior frontal gyrus, SLF: superior longitudinal fasciculus, SMG: supramarginal gyrus, STG: superior temporal gyrus. 
SLF II, the SLF III, and the SLF temporo-parietal (SLF TP) in the superficial layer, and the classical $\mathrm{AF}$ in the deep layer. ${ }^{6,39)}$ Recently, details of the terminations were disclosed by Wang et al. ${ }^{40)}$ (Fig. 1). They found a remarkable difference between the right and left hemispheres on the SLF III. The left SLF III has two branches, both of which commonly originate from the SMG. The first one terminates at the inferior part of the PreCG and the pars opercularis of the IFG, and the other one runs from the SMG dorsally, joins the SLF II, and terminates at the middle part of the PreCGand the posterior MFG. ${ }^{40)}$ The right SLF III does not have the latter branch of the left one. The SLF II originates from the angular gyrus and terminates to the middle part of the PreCG and the posterior MFG. ${ }^{40)}$

The SMG and the posterior part of the STG connected with the SLF/AF located under these cortices are profoundly associated with phonological process. Injury to this network causes conduction aphasia with characteristic symptoms of phonemic paraphasia, poor repetition, and disturbance of auditory short-term memory. ${ }^{20,36)}$

The middle-inferior part of the dominant PreCG and its subcortical area is associated with articulation and its damage result in speech apraxia or, in another word, anarthria, in which the patients have symptoms such as loss of prosody, i.e., mal-connection of phonemes and distortion of phonemes ${ }^{41,42)}$ (Fig. 2D). Damage in the anterior part of PreCG (Broadman area 6 and 4) tends to enhance loss of prosody or malconnection of phonemes more, whereas damage in the posterior part of PreCG (Broadman area 4) tends to cause distortion of phonemes. ${ }^{43)}$ Moreover, patients with "Broca's aphasia" always have the above articulatory problems with certain disturbances of the dorsal stream. ${ }^{7)}$ It is interesting that not only voice perception and process of phoneme occurs in both hemispheres as mentioned above, but also the sensorimotor transformations for speech occur bilaterally and simultaneously, according to a recent electrophysiological study. ${ }^{16)}$ The findings in the study suggest that the neural basis of language has a wider network than the one considered before, it is one that involves both hemispheres. Even though the basic phonological processes are bilateral, the computational system for higher-order language is thought to be lateralized in the dominant hemisphere. ${ }^{16)}$

\section{Driving of speech}

There is another pathway for language, associating with initiation of speech (Fig. 2E). The supplementary motor area and the medial aspect of the frontal lobe have been known to be associated with spontaneity and initiation of speech. ${ }^{44)}$ Moreover, it is well known that an injury to the SMA and/or deep frontal white matter causes transcortical motor aphasia. ${ }^{45)}$ In fact, there is an intra-frontal network connecting the medial aspect of the frontal lobe and the IFG and the middle-inferior part of PreCG, recently named as the frontal aslant tract (FAT). ${ }^{46)}$ Electrophysiological studies with direct electrical stimulation of the tract during awake surgery also suggested strong association with speech function..$^{47,48)}$ The fronto-striatal tract, running medially to the FAT from the SMA to the caudate nucleus, might also be involved in this system. ${ }^{48)}$

\section{Surgical Implications}

Neurosurgeons have traditionally believed in distinctive "the eloquent and non-eloquent" cortices in language and performed surgery based upon quite a localizationistic view. The cortical areas of Broca and Wernicke are the eloquent cortices in which tumors are unresectable, unless one can risk losing speech function, and the other cortical areas are non-eloquent cortices where surgical procedure is not supposed to cause significant speech dysfunction. Through the accumulation of experiences with electrophysiological mapping during awake surgery, it is better understood that the above central dogma is frequently not the case, especially in slow growing tumors such as low grade gliomas. ${ }^{3)}$ Low grade gliomas invading into the Broca's area can often be resected safely. ${ }^{49)}$ Recently, Saito et al. ${ }^{50)}$ reported that the frontal language area could not be identified on intraoperative brain mapping in gliomas involving the pars triangularis of the IFG frequently, suggesting that language network reorganization does occur in slow growing tumors. Tumors in the Wernicke area are also sometimes resectable when surgery is done carefully in the awake setting, thanks to plasticity of the brain and redundancy of the language network. ${ }^{51,52)}$ Moreover, even though a cortical area cannot be resected for preserving language function, if the tumor recurs, that area would no longer be functioning and, thus could be resected in a second surgery. ${ }^{53)}$

Neurosurgeons have tended to focus on preservation of the cortex and thus, surgery has been sometimes performed based on the functional information of language obtained only through cortical mapping studies with subdural electrodes, without real-time evaluation of the white matter. Since the gray and white matters are part of the same interactive process in the network, with dynamic process of language consisting of a continuous and redundant stream 
of information, ${ }^{3)}$ surgery must be performed in the awake setting, evaluating the symptoms online during surgery with attention to not only the cortex, but also the white matter, which is the substrate or backbone of the network. ${ }^{48,54)}$ For preservation of language in tumor surgery, evaluation of the white matter with electrical stimulation is no less important than evaluation of the cortex.

\section{Intraoperative Mapping of the Language Function}

For preserving language function, cortical mapping of the language, as well as subcortical mapping is mandatory under awake surgery. ${ }^{12)}$ There are two groups of neurosurgeons with respect to electrocortical mapping methods; they are called the "positive mapping" and the "negative mapping" strategies. ${ }^{12,50,55)}$ The positive mapping strategy postulates that surgeons should observe "positive findings" such as speech arrest or anomia, at least in one cortical area in order to obtain the positive control to confirm that the entire mapping result is effective. When the mapping procedure elicits symptoms, those areas resulting in negative mapping with the same stimulation protocol could be considered as truly negative and, thus judged to be safely resectable. From the viewpoint of the negative mapping strategy, which was first introduced by Sanai et al., the positive finding is not always necessary if the brain is stimulated with enough intensity. ${ }^{55)}$ It might allow a smaller craniotomy and less risk of seizure attack due to the strong stimulation intensity.

Although language has many functional aspects as described above, it is difficult to evaluate each and every aspect of language during awake surgery in a limited time that the patients are able to tolerate, thus the language tasks should be selected appropriately, depending on the patient's back ground (living environment, occupation, etc.), and the tumor location plus the relevant white matter tracts. ${ }^{12)}$ Picture naming is the most frequently used task for evaluation for all brain areas associated with language function, both cortical and subcortical, probably because it needs multiple steps of the language processing, visual recognition, semantic processing, lexical access, phonological encoding, and speech production. ${ }^{12,56)}$ Counting tasks are especially useful for speech articulation. ${ }^{12,56)}$ It is almost always possible to elicit speech arrest on the middle-inferior part of the PreCG, i.e., the ventral premotor cortex, ${ }^{12,57)}$ with either the counting or picture naming task. This finding is therefore considered to be a positive control of the mapping and the intensity of the stimulation is utilized thereafter for both cortical and subcortical mapping. ${ }^{12)}$ The auditory comprehension task is necessary, especially in the temporal and parietal lobe lesions to assess the perceptive aspects of language. ${ }^{56)}$ It also reflects the semantic processing. The repetition task is not necessarily associated with the semantic processing but mainly with the phonological processing, including the perceptive aspects of the language. It should be used when mapping in and around the SMG and the subcortical area underneath where SLF is running as the main association fiber of phonological processing. ${ }^{12)}$ The semantic aspects can be evaluated by using the pyramid and palm trees test, which are a semantic memory task, and the auditory comprehension task as described above. The IFOF, one of the main association fibers of semantic processing, is frequently encountered in the insular gliomas or the insulo-opercular gliomas with either the frontal or the temporal involvement, or both, usually at a deeper surgical field such as the white matter under the IFG or anterior insula or the temporal stem. ${ }^{12)}$ The FAT might be encountered in the frontal lobe tumors in the white matter underneath the superior frontal sulcus and middle frontal gyrus, with slowness or speech arrest as symptoms of electrical stimulation. ${ }^{47)}$

\section{Conclusion}

Exploring the neural basis of language continues worldwide. It is likely entering into a new paradigm with a drastic change in the view of the brain itself, progressing from the rigid and localizationistic to the flexible and associationistic view. ${ }^{6}$ Please note that the neural basis of language described in this article is a summary of the present knowledge and only a one-sided view with a lot of controversial points. Each neurosurgeon should keep updating their knowledge of the neural basis of language and not only use it for everyday patient care, but also play a role in the progress of neuroscience for the better care in the future, since we are the only ones who face the living human brain under direct vision.

\section{Conflicts of Interest Disclosure}

The authors have no conflict of interest regarding this article.

\section{References}

1) Brodmann K: Vergleichende lokalisationslehre der grosshirnrinde in ihren prinzipien dargestellt auf grund des zellenbaues. (Translated and edited Laurence J. Garey London: Imperial College Press, 1999). Leipzig, JA Barth, 1909 
2) Penfield W, Boldrey E: Somatic motor and sensory representation in the cerebral cortex of man as studied by electrical stimulation. Brain 60: 389-443, 1937

3) De Benedictis A, Duffau H: Brain hodotopy: from esoteric concept to practical surgical applications. Neurosurgery 68: 1709-1723; discussion 1723, 2011

4) Catani M, ffytche DH: The rises and falls of disconnection syndromes. Brain 128: 2224-2239, 2005

5) Hillis AE, Work M, Barker PB, Jacobs MA, Breese EL, Maurer K: Re-examining the brain regions crucial for orchestrating speech articulation. Brain 127: 1479-1487, 2004

6) Catani M, de Schotten MT: Atlas of Human Brain Connections. London, Oxford University Press, 2012

7) Dronkers NF, Plaisant O, Iba-Zizen MT, Cabanis EA: Paul Broca's historic cases: high resolution MR imaging of the brains of Leborgne and Lelong. Brain 130: 1432-1441, 2007

8) Penfield W, Roberts L: Speech and Brain Mechanisms. New Jersey, Princeton University Press, 1959

9) Mohr JP, Pessin MS, Finkelstein S, Funkenstein HH, Duncan GW, Davis KR: Broca aphasia: pathologic and clinical. Neurology 28: 311-324, 1978

10) Tonkonogy J, Goodglass H: Language function, foot of the third frontal gyrus, and rolandic operculum. Arch Neurol 38: 486-490, 1981

11) Hickok G, Poeppel D: Dorsal and ventral streams: a framework for understanding aspects of the functional anatomy of language. Cognition 92: 67-99, 2004

12) Fernández Coello A, Moritz-Gasser S, Martino J, Martinoni M, Matsuda R, Duffau H: Selection of intraoperative tasks for awake mapping based on relationships between tumor location and functional networks. J Neurosurg 119: 1380-1394, 2013

13) Sahin NT, Pinker S, Cash SS, Schomer D, Halgren E: Sequential processing of lexical, grammatical, and phonological information within Broca's area. Science 326: 445-449, 2009

14) Kinno R, Ohta S, Muragaki Y, Maruyama T, Sakai KL: Differential reorganization of three syntax-related networks induced by a left frontal glioma. Brain 137: 1193-1212, 2014

15) Obleser J, Eisner F, Kotz SA: Bilateral speech comprehension reflects differential sensitivity to spectral and temporal features. J Neurosci 28: 8116-8123, 2008

16) Cogan GB, Thesen T, Carlson C, Doyle W, Devinsky O, Pesaran B: Sensory-motor transformations for speech occur bilaterally. Nature 507: 94-98, 2014

17) Chang EF, Rieger JW, Johnson K, Berger MS, Barbaro NM, Knight RT: Categorical speech representation in human superior temporal gyrus. Nat Neurosci 13: 1428-1432, 2010

18) Saur D, Schelter B, Schnell S, Kratochvil D, Küpper H, Kellmeyer P, Kümmerer D, Klöppel S, Glauche V, Lange R, Mader W, Feess D, Timmer J, Weiller $\mathrm{C}$ : Combining functional and anatomical connectivity reveals brain networks for auditory language comprehension. Neuroimage 49: 3187-3197, 2010
19) Upadhyay J, Silver A, Knaus TA, Lindgren KA, Ducros M, Kim DS, Tager-Flusberg H: Effective and structural connectivity in the human auditory cortex. J Neurosci 28: 3341-3349, 2008

20) Leff AP, Schofield TM, Crinion JT, Seghier ML, Grogan A, Green DW, Price CJ: The left superior temporal gyrus is a shared substrate for auditory short-term memory and speech comprehension: evidence from 210 patients with stroke. Brain 132: 3401-3410, 2009

21) Buchsbaum RB, Hickok G, Humphries C: Role of left posterior superior temporal gyrus in phonological processing for speech comprehension and production. Cognitive Science 25: 663-678, 2001

22) Flinker A, Korzeniewska A, Shestyuk AY, Franaszczuk PJ, Dronkers NF, Knight RT, Crone NE: Redefining the role of Broca's area in speech. Proc Natl Acad Sci USA 112: 2871-2875, 2015

23) Takahashi N, Kawamura M, Shinotou H, Hirayama K, Kaga K, Shindo M: Pure word deafness due to left hemisphere damage. Cortex 28: 295-303, 1992

24) Price CJ: The anatomy of language: a review of 100 fMRI studies published in 2009. Ann N Y Acad Sci 1191: 62-88, 2010

25) Schwartz MF, Kimberg DY, Walker GM, Faseyitan O, Brecher A, Dell GS, Coslett HB: Anterior temporal involvement in semantic word retrieval: voxel-based lesion-symptom mapping evidence from aphasia. Brain 132: 3411-3427, 2009

26) Glasser MF, Rilling JK: DTI tractography of the human brain's language pathways. Cereb Cortex 18: 2471-2482, 2008

27) Makris N, Papadimitriou GM, Kaiser JR, Sorg S, Kennedy DN, Pandya DN: Delineation of the middle longitudinal fascicle in humans: a quantitative, in vivo, DT-MRI study. Cereb Cortex 19: 777-785, 2009

28) Maldonado IL, de Champfleur NM, Velut S, Destrieux C, Zemmoura I, Duffau H: Evidence of a middle longitudinal fasciculus in the human brain from fiber dissection. J Anat 223: 38-45, 2013

29) Spitsyna G, Warren JE, Scott SK, Turkheimer FE, Wise RJ: Converging language streams in the human temporal lobe. J Neurosci 26: 7328-7336, 2006

30) Tyler LK, Marslen-Wilson W: Fronto-temporal brain systems supporting spoken language comprehension. Philos Trans R Soc Lond, B, Biol Sci 363: 1037-1054, 2008

31) Tyler LK, Marslen-Wilson WD, Randall B, Wright P, Devereux BJ, Zhuang J, Papoutsi M, Stamatakis EA: Left inferior frontal cortex and syntax: function, structure and behaviour in patients with left hemisphere damage. Brain 134: 415-431, 2011

32) Saur D, Kreher BW, Schnell S, Kümmerer D, Kellmeyer P, Vry MS, Umarova R, Musso M, Glauche V, Abel S, Huber W, Rijntjes M, Hennig J, Weiller C: Ventral and dorsal pathways for language. Proc Natl Acad Sci USA 105: 18035-18040, 2008

33) Sarubbo S, De Benedictis A, Maldonado IL, Basso G, Duffau H: Frontal terminations for the inferior fronto-occipital fascicle: anatomical dissection, DTI 
study and functional considerations on a multicomponent bundle. Brain Struct Funct 218: 21-37, 2013

34) Grodzinsky Y, Santi A: The battle for Broca's region. Trends Cogn Sci (Regul Ed) 12: 474-480, 2008

35) Binder JR, Desai RH, Graves WW, Conant LL: Where is the semantic system? A critical review and meta-analysis of 120 functional neuroimaging studies. Cereb Cortex 19: 2767-2796, 2009

36) Fridriksson J, Kjartansson O, Morgan PS, Hjaltason H, Magnusdottir S, Bonilha L, Rorden C: Impaired speech repetition and left parietal lobe damage. J Neurosci 30: 11057-11061, 2010

37) Roux FE, Lubrano V, Lauwers-Cances V, Trémoulet M, Mascott CR, Démonet JF: Intra-operative mapping of cortical areas involved in reading in mono- and bilingual patients. Brain 127: 1796-1810, 2004

38) Maldonado IL, Moritz-Gasser S, Duffau H: Does the left superior longitudinal fascicle subserve language semantics? A brain electrostimulation study. Brain Struct Funct 216: 263-274, 2011

39) Kamali A, Sair HI, Radmanesh A, Hasan KM: Decoding the superior parietal lobule connections of the superior longitudinal fasciculus/arcuate fasciculus in the human brain. Neuroscience 277: 577-583, 2014

40) Wang X, Pathak S, Stefaneanu L, Yeh FC, Li S, Fernandez-Miranda JC: Subcomponents and connectivity of the superior longitudinal fasciculus in the human brain. Brain Struct Funct 2015 [Epub ahead of print]

41) Kasahata N: Speech disturbances due to left precentral cortical lesions. Neurocase 20: 328-337, 2014

42) Tate MC, Herbet G, Moritz-Gasser S, Tate JE, Duffau $\mathrm{H}$ : Probabilistic map of critical functional regions of the human cerebral cortex: Broca's area revisited. Brain 137: 2773-2782, 2014

43) Otsuki M: Symptomatology of anarthria. Japanese Journal of Neuropsychology 21: 172-182, 2005

44) Alario FX, Chainay H, Lehericy S, Cohen L: The role of the supplementary motor area (SMA) in word production. Brain Res 1076: 129-143, 2006

45) Morgan VL, Mishra A, Newton AT, Gore JC, Ding Z: Integrating functional and diffusion magnetic resonance imaging for analysis of structure-function relationship in the human language network. PLoS ONE 4: e6660, 2009

46) Catani M, Dell'acqua F, Vergani F, Malik F, Hodge H, Roy P, Valabregue R, Thiebaut de Schotten M: Short frontal lobe connections of the human brain. Cortex 48: 273-291, 2012

47) Fujii M, Maesawa S, Motomura K, Futamura M, Hayashi Y, Koba I, Wakabayashi T: Intraoperative subcortical mapping of a language-associated deep frontal tract connecting the superior frontal gyrus to
Broca's area in the dominant hemisphere of patients with glioma. J Neurosurg 122: 1390-1396, 2015

48) Kinoshita M, de Champfleur NM, Deverdun J, Moritz-Gasser S, Herbet G, Duffau H: Role of frontostriatal tract and frontal aslant tract in movement and speech: an axonal mapping study. Brain Struct Funct 220: 3399-3412, 2015

49) Duffau H: The "frontal syndrome" revisited: lessons from electrostimulation mapping studies. Cortex 48: 120-131, 2012

50) Saito T, Muragaki Y, Maruyama T, Tamura M, Nitta M, Tsuzuki S, Konishi Y, Kamata K, Kinno R, Sakai $\mathrm{KL}$, Iseki $\mathrm{H}$, Kawamata T: Difficulty in identification of the frontal language area in patients with dominant frontal gliomas that involve the pars triangularis. $J$ Neurosurg 1-9, 2016 [Epub ahead of print]

51) Sarubbo S, Le Bars E, Moritz-Gasser S, Duffau H: Complete recovery after surgical resection of left Wernicke's area in awake patient: a brain stimulation and functional MRI study. Neurosurg Rev 35: 287-292; discussion 292, 2012

52) Yogarajah M, Focke NK, Bonelli SB, Thompson P, Vollmar C, McEvoy AW, Alexander DC, Symms MR, Koepp MJ, Duncan JS: The structural plasticity of white matter networks following anterior temporal lobe resection. Brain 133: 2348-2364, 2010

53) Duffau H, Taillandier L: New concepts in the management of diffuse low-grade glioma: proposal of a multistage and individualized therapeutic approach. Neuro-oncology 17: 332-342, 2015

54) Duffau H: New concepts in surgery of WHO grade II gliomas: functional brain mapping, connectionism and plasticity-a review. J Neurooncol 79: 77-115, 2006

55) Sanai N, Mirzadeh Z, Berger MS: Functional outcome after language mapping for glioma resection. $N$ Engl J Med 358: 18-27, 2008

56) Kayama T; Guidelines committee of the Japan awake surgery conference: The guidelines for awake craniotomy guidelines committee of the Japan awake surgery conference. Neurol Med Chir (Tokyo) 52: 119-141, 2012

57) Chang EF, Breshears JD, Raygor KP, Lau D, Molinaro AM, Berger MS: Stereotactic probability and variability of speech arrest and anomia sites during stimulation mapping of the language dominant hemisphere. $J$ Neurosurg 1-8, 2016 [Epub ahead of print]

Address reprint requests to: Masazumi Fujii, MD, PhD, Department of Neurosurgery, Fukushima Medical University, 1 Hikarigaoka, Fukushima, Fukushima 960-1295 Japan. e-mail: fujiim@fmu.ac.jp 